\title{
Proceedings of the Canadian Frailty Network Summit: Medication Optimization for Frail Older Canadians, Toronto, Monday April 24, 2017

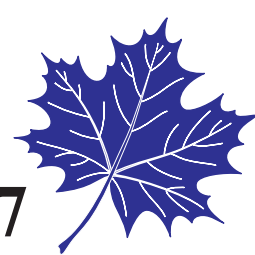

John Muscedere, MD, FRCPC ${ }^{1,2}$, Perry Kim, $\mathrm{PhD}^{2}$, Peter Aitken, $\mathrm{BBM}^{3}$, Michael Gaucher, MBA ${ }^{4}$, Robin Osborn, $\mathrm{MBA}^{5}$, Barbara Farrell, BPharm, PharmD, FCSHP 6 , Jayna Holroyd-Leduc, MC, FRCPC ${ }^{7}$, Laurie Mallery, MD, FRCPC ${ }^{8}$, Henry Siu, MD, MSc, CCFP(COE) ${ }^{9}$, James Downar, MDCM, MHSc, FRCPC ${ }^{10}$, Todd C. Lee, MD, MPH, FRCPC ${ }^{11}$, Emily McDonald, MD, MSc, FRCPC ${ }^{11}$, Lisa Burry, BPharm, PharmD ${ }^{12,10}$

${ }^{1}$ Department of Critical Care Medicine, Queen's University and ${ }^{2}$ Canadian Frailty Network, Kingston, ON, Canada; ${ }^{3}$ Partnerships, Development and Government Relations, Canadian Frailty Network, Kingston, ON, Canada;

${ }^{4}$ Pharmaceuticals and Health Workforce Information Services, Canadian Institute of Health Information, Ottawa, ON, Canada; ${ }^{5}$ International Health Policy and Practice Innovations for the Commonwealth Fund, New York, NY, U.S.A.;

${ }^{6}$ Bruyère Research Institute, Department of Family Medicine, University of Ottawa, Ottawa, ON, Canada; ${ }^{7}$ Medicine and Community Health Science, University of Calgary, Calgary, AB, Canada; ${ }^{8}$ Geriatric Medicine, Dalhousie University, Halifax, NS, Canada; ${ }^{9}$ Family Medicine at McMaster University, Hamilton, ON, Canada; ${ }^{10}$ Department of Medicine, University of Toronto, Toronto, ON, Canada; ${ }^{11}$ Mc Gill University Health Centre, Montreal, HQ, Canada; ${ }^{12}$ Mount Sinai Hospital and the University of Toronto, Toronto, ON, Canada

DOI:https://doi.org/10.5770/cgj.20.293

\begin{abstract}
Appropriate and optimal use of medication and polypharmacy are especially relevant to the care of older Canadians living with frailty, often impacting their health outcomes and quality of life. A majority (two thirds) of older adults (65 or older) are prescribed five or more drug classes and over one-quarter are prescribed 10 or more drugs. The risk of adverse drug-induced events is even greater for those aged 85 or older where $40 \%$ are estimated to take drugs from 10 or more drug classes. The Canadian Frailty Network (CFN), a pan-Canadian non-for-profit organization funded by the Government of Canada through the Networks of Centres of Excellence Program (NCE), is dedicated to improving the care of older Canadian living with frailty and, as part of its mandate, convened a meeting of stakeholders from across Canada to seek their perspectives on appropriate medication prescription. The CFN Medication Optimization Summit identified priorities to help inform the design of future research and knowledge mobilization efforts to facilitate optimal medication prescribing in older adults living with frailty. The priorities were developed and selected through a modified Delphi process commencing before and concluding during the summit. Herein we describe the overall approach/process to the summit, a summary of all the presentations and discussions, and the top ten priorities selected by the participants.
\end{abstract}

Key words: frailty, seniors, polypharmacy, deprescribing, medication optimization, older adults

\section{INTRODUCTION}

With increasing age, the prevalence of chronic medical conditions increases and subsequently the number of medications that someone is likely to be prescribed increases. ${ }^{(1,2)}$ It is estimated that nearly two-thirds of seniors older than 65 are prescribed five or more drug classes and more than one-quarter are prescribed 10 or more. ${ }^{(3)}$ In those over the age of 85 , approximately $40 \%$ take 10 or more drug classes. ${ }^{(1)}$ In the elderly, polypharmacy has been associated with poor outcomes including cognitive impairment, falls, hospitalization, and mortality. ${ }^{(4,5)}$ A particularly vulnerable population is those living with frailty, where polypharmacy has been associated with a higher propensity for drug-drug interactions, drug-disease interactions, and adverse reactions including mortality. ${ }^{(6,7,8)}$ Inappropriate prescribing can increase vulnerability to the development of frailty. ${ }^{(9,10)}$ The risk of polypharmacy may be underappreciated, as there are few studies of drug-drug interactions in those receiving numerous drugs, and limited information on the pharmacodynamics of medications in older adults living with frailty who may have altered drug clearance because of age-related changes and increased prevalence of end-organ disease such as chronic liver and kidney disease. ${ }^{(2)}$ Overall, there is a need to re-examine prescribing practices for those in late life living with frailty. 
The Canadian Frailty Network (CFN) is a pan-Canadian non-for-profit organization funded by the Government of Canada through the Networks of Centres of Excellence Program (NCE) whose mission is to improve the care of those living with frailty in Canada. To inform initiatives addressing appropriate medication prescription in those with frailty, CFN convened a meeting of stakeholders from across Canada to seek their perspectives as part of a broad consultation process. The CFN Medication Optimization summit identified priorities to help inform the design of future research and knowledge mobilization efforts to facilitate optimal medication prescribing in frail adults. Herein we report on the priorities identified at the summit, which provides timely information for research funders, clinicians, and decisionmakers. The term "optimization" was chosen as an inclusive term, which includes related issues such as "polypharmacy", "appropriate prescribing", and "deprescribing".

\section{SELECTION OF PARTICIPANTS}

In preparation for the summit, Canadian stakeholders and opinion leaders were identified using the following criteria: they were investigators on $\mathrm{CFN}$-funded research grants studying appropriate medication prescription, had published in peer-reviewed literature on the topic, were in relevant decision-making roles, or were citizens/family caregivers affected by frailty. Once identified, they were approached for participation and, as a condition of participation, were asked to abide with a consensus-building process. Fifty-three delegates attended, including researchers, clinicians (e.g., physicians, pharmacists), health-care administrators, policy experts, public association representatives, and citizens (Table 1).

\section{DELPHI PROCESS}

A modified Delphi process was used to rank priorities prior to the meeting to guide discussion during the in-person meeting.
(11) Items for inclusion into the process were selected by asking each participant to identify their top two priorities during the registration process. In addition, a request was distributed to all members of the CFN mailing list (approx. 3,000) including researchers and stakeholders from across Canada, to identify their top priorities through the CFN website (www.cfn-nce. ca). The attendees were then asked to rate the initial list of 27 priorities (Appendix 1) using a nine-point Likert scale. During the first round of the Delphi process, attendees also had the option of adding additional priorities. A total of three rounds were conducted, where the bottom seven were removed during each round and the remaining were ranked again. By the third round, the top 10 rank priorities had stabilized (Table 2). During the face-face meeting, the participants were live-polled ${ }^{(12)}$ and the priorities were ranked (again by a nine-point scale) to narrow the list to the top eight priorities that then formed the basis for discussion (Table 2). Initial comments from the delegates pointed out that the priorities included both research and knowledge mobilization activities, and that the distribution of scores was narrow within the rankings. Summit participants were then provided with an overview of the current issues related to medication prescribing for frail elderly and snapshots of currently CFN-funded research on medication optimization, before re-ranking the priorities.

\section{CURRENT UNDERSTANDING OF MEDICATION OPTIMIZATION FOR THE FRAIL ELDERLY - PANEL DISCUSSION}

A panel representing a variety of stakeholder groups presented current understanding and issues for medication optimization in older adults living with frailty. First, an overview of Canadian databases tracking medication prescription in the elderly was provided by Michael Gaucher. The Canadian Institute for Health Information (CIHI) maintains two databases: National Prescription Drug Utilization Information System (NPDUIS), and the Continuing Care Reporting System (CCRS).

TABLE 1.

CFN medication summit delegate background

\begin{tabular}{lccc}
\hline \multicolumn{1}{c}{ Background } & $n(\%)$ & HealthCare Sector & $n(\%)$ \\
\hline Academic/Researcher & $25(47.2)$ & University or research institute & $20(37.7)$ \\
Health-care Professional & $14(26.4)$ & No response & $8(15.1)$ \\
Administrator & $4(7.5)$ & Professional/advocacy/Not-for-profit organization & $7(13.2)$ \\
Policy/Decision-Maker & $4(7.5)$ & Hospital & $6(11.3)$ \\
Frail Older Adult or Caregiver & $2(3.8)$ & Residential/long-term care provider & $5(9.4)$ \\
Quality/Patient Safety & $2(3.8)$ & Government/health ministry & $3(5.7)$ \\
Funding Agency & $2(3.8)$ & Home/community care provider & $2(3.8)$ \\
& & Industry-pharmaceutical company & $1(1.9)$ \\
& & Industry-retail pharmacy & $1(1.9)$ \\
\hline
\end{tabular}


NPDUIS ${ }^{(13)}$ provides prescription-claims-level longitudinal data (representing $70 \%$ of Canadian seniors), while $\mathrm{CCRS}^{(14)}$ provides utilization information on continuing care services in Canadian hospitals and long-term care homes. A 2012 report utilizing these databases found that $25 \%$ of seniors $(65+)$ had claims for ten or more drug classes; the proportion of those 85 years and older using ten or more drugs (39.3\%) is double those 65 to $85(20.0 \%){ }^{(3)}$ Roughly $40 \%$ of seniors use at least one potentially inappropriate drug, based on Beers criteria. ${ }^{(15,16)} \mathrm{A}$ forthcoming report will examine polypharmacy based on care

TABLE 2

Top ten medication optimization priorities identified from round three of Delphi process, and top eight medication optimization priorities as identified from round four of the Delphi process from live vote by summit delegates

\begin{tabular}{|c|c|c|c|c|c|}
\hline $\begin{array}{l}\text { Round } \\
\quad 3 \\
\text { Rank }\end{array}$ & $\begin{array}{l}\text { Likert } \\
\text { Score } \\
(x / 9)\end{array}$ & Priority & $\begin{array}{l}\text { Round } \\
\quad 4 \\
\text { Rank }\end{array}$ & $\begin{array}{c}\text { Score } \\
(x / 9)\end{array}$ & Priority \\
\hline 1 & 8.06 & $\begin{array}{l}\text { Building an (electronic) system and practice system } \\
\text { so that a patient's medications can be monitored and } \\
\text { reviewed in real time to determine the current need } \\
\text { and appropriateness of each medication, as well as } \\
\text { potential for adverse drug-drug interactions. }\end{array}$ & 1 & 7.6 & $\begin{array}{l}\text { Building an (electronic) system and practice system } \\
\text { so that a patient's medications can be monitored and } \\
\text { reviewed in real time to determine the current need } \\
\text { and appropriateness of each medication, as well as } \\
\text { potential for adverse drug-drug interactions. }\end{array}$ \\
\hline 2 & 7.94 & $\begin{array}{l}\text { Improving efforts (e.g., funding) towards developing } \\
\text { novel technologies focused on facilitating appropriate } \\
\text { medication prescribing and/or deprescribing in older } \\
\text { adults living with frailty. }\end{array}$ & 2 & 6.9 & $\begin{array}{l}\text { Increasing research into developing or improving } \\
\text { models that facilitate pharmacists playing an active } \\
\text { role in the process of monitoring and assessing use } \\
\text { of potentially inappropriate medications. }\end{array}$ \\
\hline 3 & 7.61 & $\begin{array}{l}\text { Increasing research into developing or improving } \\
\text { models that facilitate pharmacists playing an active } \\
\text { role in the process of monitoring and assessing use } \\
\text { of potentially inappropriate medications. }\end{array}$ & 3 & 6.7 & $\begin{array}{l}\text { Improving efforts (e.g., funding) towards developing } \\
\text { novel technologies focused on facilitating appropriate } \\
\text { medication prescribing and/or deprescribing in older } \\
\text { adults living with frailty. }\end{array}$ \\
\hline 4 & 7.56 & $\begin{array}{l}\text { Advocacy for clinical trials that specifically evaluate } \\
\text { concomitant multiple medication use and the } \\
\text { potential for adverse drug-drug interactions specific } \\
\text { to seniors living with frailty. }\end{array}$ & 4 & 6.1 & $\begin{array}{l}\text { Promoting and supporting the development of a nation- } \\
\text { wide Pharmacare plan for all Canadians, which would } \\
\text { disproportionately benefit older adults in late life since } \\
\text { this group is likely to be taking more medications. }\end{array}$ \\
\hline 5 & 7.06 & $\begin{array}{l}\text { Further research into the values and preferences held } \\
\text { by elderly patients living with frailty with respect to } \\
\text { medication use (e.g., determine perspectives regarding } \\
\text { appropriate medication prescription or deprescribing). }\end{array}$ & 4 & 6.1 & $\begin{array}{l}\text { New technologies being developed so that patients } \\
\text { (and their trained care providers) are empowered to } \\
\text { monitor and assess their own medication use. }\end{array}$ \\
\hline 6 & 7.0 & $\begin{array}{l}\text { Further research into best practices for shared } \\
\text { decision making with respect to medication use by } \\
\text { seniors living with frailty. [eliminated in round four } \\
\text { live vote] }\end{array}$ & 5 & 6.0 & $\begin{array}{l}\text { Advocacy for clinical trials that specifically evaluate } \\
\text { concomitant multiple medication use and the } \\
\text { potential for adverse drug-drug interactions specific } \\
\text { to seniors living with frailty. }\end{array}$ \\
\hline 7 & 7.0 & $\begin{array}{l}\text { All new registration trials for medications targeted } \\
\text { at older individuals include and report on the } \\
\text { differential impact on individuals living with frailty. } \\
\text { [eliminated in round four live vote] }\end{array}$ & 5 & 6.0 & $\begin{array}{l}\text { Further research into the values and preferences held } \\
\text { by elderly patients living with frailty with respect to } \\
\text { medication use (e.g., determine perspectives regarding } \\
\text { appropriate medication prescription or deprescribing). }\end{array}$ \\
\hline 8 & 7.0 & $\begin{array}{l}\text { Promoting and supporting the development of a nation- } \\
\text { wide Pharmacare plan for all Canadians, which would } \\
\text { disproportionately benefit older adults in late life since } \\
\text { this group is likely to be taking more medications. }\end{array}$ & 6 & 6.0 & $\begin{array}{l}\text { Advocacy for clinical trials that specifically evaluate } \\
\text { the therapeutic benefit and adverse effects of new } \\
\text { and current medications on seniors living with frailty }\end{array}$ \\
\hline 9 & 7.0 & $\begin{array}{l}\text { New technologies being developed so that patients } \\
\text { (and their trained care providers) are empowered to } \\
\text { monitor and assess their own medication use. }\end{array}$ & & & \\
\hline 10 & 6.83 & $\begin{array}{l}\text { Advocacy for clinical trials that specifically evaluate } \\
\text { the therapeutic benefit and adverse effects of new } \\
\text { and current medications on seniors living with frailty }\end{array}$ & & & \\
\hline
\end{tabular}


location (including long-term care) and income, inappropriate drug use and adverse drug reactions amongst seniors, and prescribing patterns by drug class.

Robin Osborn highlighted points from the Commonwealth 2014 Health Policy survey in 11 countries. ${ }^{(17)}$ Medication costs can be a barrier to appropriate medication utilization. Due to cost, $8 \%$ of elderly patients in Canada and $16 \%$ in the United States do not take medications as prescribed (compared to 3\% in France). The survey data also indicate that $34 \%$ of older $(65+)$ Canadians are taking four or more medications (almost $50 \%$ in the US). Among older Canadians taking two or more medications, almost 1 in 5 did not have a medication review in the past year (slightly less in the US). When prescribed new medications, more than 1 in 4 older Canadians did not have a discussion with the provider about potential side effects, and the same proportion did not have a medication reconciliation conducted upon discharge from hospital.

In regard to using technology to facilitate medication optimization, Osborn identified two examples: "Patients Like Me" and "Proteus Discover". "Patients Like Me" is a free online information sharing resource with 500,000 members that share information and track/report their health status on 30-40 chronic conditions, using a safety monitoring platform. ${ }^{(18)}$ Captured data include numerous dimensions, such as, patient reported outcomes, side-effects, adherence/ non-adherence, and cross-conditional information. Proteus Discover is a digestible biomarker that can be put on a pill, which allows physicians to track adherence and response to treatment. ${ }^{(19)}$ This product is in the process of clinical testing within the United States. ${ }^{(20)}$ Osborn closed by discussing a collaborative involving 15 major US delivery systems (approximately 50 million patients), which seeks international solutions as part of their process. ${ }^{(21)}$ As an example of cross-nation learning, six American health systems in the collaborative have already adopted the deprescribing model developed in Canada by Barbara Farrell. (22)

Dr. Barbara Farrell then highlighted the importance of deprescribing. In her practice, it is not unusual to see patients taking 25 to 30 medications - a situation that has anecdotally increased during the past two decades. Problems associated with polypharmacy include: lack of patient understanding/ knowledge about medications (including the reason the medication was prescribed), unintentional non-compliance (e.g., forgetting, confusion), intentional non-compliance based on pill burden, and prescribing cascades (medications prescribed to address side effects from other medications). In addition, large numbers of medications can contribute to impaired balance, falls, and cognitive impairment for older adults living with frailty, all of which are often reversible once the medications are halted or tapered.

Farrell identified several systematic barriers to deprescribing solutions and strategies including: difficulty determining the original rationale for the prescription and duration; determining the effectiveness and safety of the medication; and gauging relevance to current care goals for older adults living with frailty. These issues are compounded by a lack of provider awareness about polypharmacy and limited understanding of how medications can contribute to symptoms. Compounding the inertia associated with halting medications is the poor understanding of the necessary conditions to stop or alter medications, and how to effectively monitor the stoppage/reduction. Further, providers tend to

TABLE 3

Rank of the top medication optimization priorities identified from final round of Delphi process (live vote)

\begin{tabular}{|c|c|c|}
\hline $\begin{array}{l}\text { Final } \\
\text { Rank }\end{array}$ & $\begin{array}{l}\text { Score } \\
(x / 9)\end{array}$ & Priority \\
\hline 1 & 7.3 & $\begin{array}{l}\text { Improving efforts (e.g., funding) towards developing novel technologies innovations focused on facilitating appropriate } \\
\text { medication prescribing and/or deprescribing in older adults living with frailty. }\end{array}$ \\
\hline 2 & 6.5 & $\begin{array}{l}\text { Increasing research into developing or improving models that facilitate pharmacists playing an active role in the process } \\
\text { of monitoring and assessing use of potentially inappropriate medications. }\end{array}$ \\
\hline 2 & 6.5 & $\begin{array}{l}\text { Further research into the values and preferences held by elderly patients living with frailty with respect to medication } \\
\text { use (e.g., determine perspectives regarding appropriate medication prescription or deprescribing). }\end{array}$ \\
\hline 3 & 6.4 & $\begin{array}{l}\text { Building an (electronic) system and practice system so that a patient's medications can be monitored and reviewed in } \\
\text { real time to determine the current need and appropriateness of each medication, as well as potential for adverse drug- } \\
\text { drug interactions. }\end{array}$ \\
\hline 3 & 6.4 & $\begin{array}{l}\text { Advocacy for clinical trials that specifically evaluate the therapeutic benefit and adverse effects of new and current } \\
\text { medications on seniors living with frailty. [merged and edited] }\end{array}$ \\
\hline 4 & 6.2 & $\begin{array}{l}\text { New innovations being developed so that patients (and their trained care providers) are empowered to monitor and } \\
\text { assess their own medication use. }\end{array}$ \\
\hline 5 & 4.9 & Promoting and supporting the development of a nation-wide Pharmacare plan for all Canadians. \\
\hline
\end{tabular}


be reluctant to halt a medication that another provider has prescribed. Farrell concluded with several recommendations to facilitate deprescribing and optimal medication use:

- Better documentation regarding the rationale for prescribing a medication, the proposed duration, and re-assessment guidance;

- More use of shareable electronic medical records (EMRs) to inform those who contemplate medication rationalization;

- Limitations on the duration of prescription for certain types/classes of medication;

- Better education regarding polypharmacy and deprescribing for health-care providers and the public;

- Need for better education and data about pharmacokinetics and pharmacodynamics in the elderly;

- Mandatory periodic medication reviews (e.g., every 6 months) for patients over the age of 85 , by a qualified health-care professional;

- More research focused on deprescribing, including randomized controlled trials (RCT);

- Development of clinical practice guidelines for deprescribing; and

- Education of all stakeholders on the limitations of medications in older adults living with frailty.

Finally, Dr. Jayna Holroyd-Leduc suggested that delivering optimal medication to older adults living with frailty is not just a prescriber/provider issue, but an issue stemming from patients who often see medications as a ready solution for their conditions. Therefore, education of both providers and patients is essential. She added that prescribing should be individualized at the beginning of any treatment, with consideration given to the patient's goals of care. She also recommended that care strategies should include avoiding medications to manage conditions that can be effectively managed by behavioural therapies (e.g., sleep issues, urinary incontinence), but noted that system change needs to occur to allow time/compensation for this kind of care. Holroyd-Leduc concluded by saying that prescribers need to periodically reevaluate ongoing need for medications. Furthermore, precision medicine may better direct prescribing in the future, as many medication prescribing recommendations are currently based on average effectiveness in a select population, and therefore effectiveness which may not be universally applicable.

The ensuing discussion focused on the under-treatment of the frail elderly secondary to therapeutic nihilism. The complexities and facets of this issue underscore a need to shift thinking from "deprescribing" to "appropriate prescribing". Access to clinical information was also discussed, particularly among pharmacists. The audience agreed on the need for updated policies and strategies to provide relevant healthcare professionals across the care continuum with access to detailed drug prescription history for patients. It was finally suggested that Health Canada should mandate that frailty be measured in all drug trials to assess the differential impact in this population. The same policy approach was recommended for funders of research.

\section{OVERVIEW OF RELEVANT CFN-FUNDED RESEARCH}

Five investigators briefed the audience on examples of CFNfunded pilot studies of medication use in older adults living with frailty. Dr. Laurie Mallery presented the results from a knowledge synthesis grant for antidepressant use in long-term care (LTC) residents. She noted that while STOPP/START and Beers' criteria have been used to address polypharmacy, the majority of adverse drug events (ADEs) are attributable to "non-list" medications, likely because the list strategy does not contextualize commonly drugs used for those living with frailty. ${ }^{(23)}$ For instance, in contrast to the common belief that older adults are underdiagnosed and undertreated for depression, more than half of LTC residents are prescribed antidepressants, possibly due to overlapping symptoms between depression and frailty. Based on a systematic review and meta-analysis, the group concluded that, compared to placebo, antidepressants are not likely to improve depression for frail older adults, with and without dementia, although there may be individual patients who might benefit from treatment. Compared to placebo, side effects were significantly more frequent in those taking antidepressants and may be more serious with frailty. In addition, the knowledge synthesis project conducted by Dr. Mallery also reviewed other common chronic conditions (diabetes, hypertension, dyslipidemia) where current guidelines may not be appropriate to guide treatment decisions for patients living with frailty.

Dr. Henry Siu was the next presenter. He outlined his study of an electronic device (the eDosette) that is able to capture and provide information about when a patient takes their medications and report any side effects experienced to their family doctor and pharmacist. ${ }^{(24)}$ The eDosette was developed to assist primary care clinicians in addressing and managing polypharmacy in community-dwelling seniors. A patient stores their blister pack or dosette in the eDosette, which serially captures image data of the stored blister pack or dosette. By doing so, the eDosette can create a patient medication administration record (MAR) that can be reviewed by the pharmacist, physician, and any other member of the patient's health-care team. The current pilot study that was described examined the eDosette in 60 community dwelling seniors managing their own medications from four different primary care practices across three family health teams in Ontario. Siu also suggested several priorities/opportunities for CFN to consider. First, medication optimization must be primary care-centred and that CFN should consider prioritizing projects that involve primary care and include primary carerelevant outcomes. Second, he suggested that CFN prioritize applications that incorporate a communication strategy about medications, especially those that demonstrate ways to bridge 
different practice settings and silos, and between primary and specialist care. Third, CFN should encourage partnerships with industry partners to leverage academic activities, such as technology industry partners that can aid in medication optimization. Lastly, he indicated that future work in medication optimization should begin to focus on medication concordance rather than pure medication adherence. ${ }^{(25)}$

Dr. James Downar presented next on a pilot study investigating a system change to improve care for the seriously ill and frail elderly, which included a team-based approach to the review ("rationalization") of medications in hospitalized seniors using guidelines of potentially inappropriate medications from Beers', STOPP, and Choosing Wisely Canada. Overall, the team made a total of 263 recommendations, with at least one recommendation for $52 / 54$ patients (96\%). Of these recommendations, $90 \%$ were accepted by both the attending team and the patient/substitute decision-maker. These recommendations resulted in the discontinuation of 162 medications (mean 3.1 per patient), dose changes for 48 medications (mean 0.9 per patient), and the addition of 13 medications (mean 0.2 per patient). Downar concluded that the study demonstrated the critical role of pharmacists in medication rationalization, which is distinct from medication reconciliation. Future work in this area will include studying means of automating the approach.

Dr. Emily McDonald presented her work with Dr. Todd Lee evaluating the impact of an electronic deprescribing tool, MedSafer, on the reduction of potentially inappropriate medications (PIMs) as compared to usual care, in hospitalized older adults. McDonald pointed out that polypharmacy (10 plus drugs) is seen in at least 1 in 5 community-dwelling older adults and more than half of older hospitalized patients, and that older adults living with frailty are at the highest risk of adverse events due to polypharmacy. The MedSafer application is designed to cross-reference patient medical conditions with rule sets of PIMs from Beers', STOPP, and Choosing Wisely Canada. The MedSafer output yields individualized deprescribing opportunities to the treating medical team, based on a drug's likelihood of causing harm and its potential to reduce future illness, and balanced against the likelihood to improve symptoms. MedSafer was successfully tested on over 1,000 hospitalized patients across three Canadian hospital centres; 603 patients consented for a structured post-hospitalization follow-up phone interview to determine if stopping PIMs had a positive impact on their quality of life, sleep, or number of adverse drug events. Initial results show that MedSafer worked extremely well for proton pump inhibitors (PPIs) and full analysis of data is underway.

Dr. Lisa Burry presented on improving the management of sedation, pain, and delirium during hospitalization and transition to home, and highlighted a knowledge synthesis project on the utilization of antipsychotics that informed this work. The review and meta-analysis was done in collaboration with Cochrane ${ }^{(26)}$ and demonstrated that antipsychotics are not associated with a significant change in duration of delirium, mortality rate or occurrence of adverse events. The team was unable to find data specific to an older or older frail population. This $\mathrm{CFN}$-funded project led to a CIHR-funded systematic review and meta-analysis examining all drugs and strategies to treat delirium in patients in the intensive care unit (ICU). Findings have shown great variations in outcomes. Other findings include that the type of drug exposure predicts delirium. Specifically, opioids do not increase the risk of delirium, whereas undertreatment of pain and using benzodiazepines does. Burry concluded by stating that there are many repercussions of deprescribing. For instance, the discontinuation of one drug may lead to the prescribing of a second equally inappropriate drug. Burry also emphasized the need for a medication optimization processes, especially for critically ill patients during their transition out of the ICU.

\section{OPEN DISCUSSION}

Open discussion followed and focused on several topics, including:

1. The low adoption and cross-platform integration of technology as a potential facilitator/barrier to medication optimization. There are currently several technologybased systems in practice, but very few can communicate with each other.

2. The need for improved interprofessional and crosssite communication as essential for addressing the reluctance to alter medication prescribing practices, and that deprescription efforts often lead to reactive changes which should be avoided.

3. The possibility of mandating medication optimization as a standard practice/behaviour, although it was recognized that there are many potential difficulties in adopting this approach.

4. The need for increased collaboration to improve how frailty is addressed, including the need for multidisciplinary guidelines designed to specifically address the complex and unique needs of frail older adults with complex comorbidities.

\section{FINAL RANKING OF PRIORITIES}

In the last phase of the meeting, attendees were divided into eight groups with each assigned to discuss the remaining eight priorities identified during the Delphi process; they were tasked with identifying and reporting back on the key aspects of each priority and potential related challenges. Based on the recommendation of the groups and with consent of the entire audience, the two initial priorities regarding clinical trials were merged for the purposes of final voting. A live vote was then held to establish a final ranking. The group reports are summarized below by priority. 
Priority: Advocacy for clinical trials that specifically evaluate the therapeutic benefit and adverse effects of new and current medications on seniors living with frailty.

This group reported on the need to redesign or augment clinical trials to study therapeutic benefit in frail older patients, both in peer-reviewed and industry-sponsored research. Two key points were suggested: 1) To inform the design and implementation of trials, CFN could have a more substantial role in providing definitions of frailty and guidance on the unique aspects of this population to inform the design and implementation of these trials; and 2) Ways to ensure that this occurs needs to be explored, including advocating that frailty needs to be measured where appropriate for Health Canada registration and common drug review.

Priority: The development of new technologies allowing patients and their trained care providers to be empowered to track, monitor, and assess their own medications.

The group identified this priority as having great value. For example, allowing patients to carry information about their medication for when they seek medical attention would empower patients to have a better understanding of, and be more proactive in, their care. Personal banking/finances were used as an analogy, which can be accessed via a smartphone or computer. This type of technology could monitor for adverse health events, which could inform the health-care provider. Further, these technologies could be combined with wearable devices, which are still in their infancy for health-care use.

Priority: Promoting and supporting the development of a nation-wide Pharmacare plan for all Canadians, which would disproportionately benefit older adults in late life since this group is likely to be taking more medications.

A national formulary, through its buying power, could provide lower cost pharmaceuticals for the whole country and might serve to equalize regional disparities, access, and portability. This would also benefit many of the other priorities on the list, including a nationwide data platform to metrics, including appropriateness and variation of care. Also, if this option were available to younger frail individuals, it may aid in delaying the progression of frailty and development of adverse events. The group concluded that the plan should have an information system, allowing for tracking of patterns and outcomes to inform patients and providers about optimal medication use.

Priority: Further research into the values and preferences held by elderly patients living with frailty with respect to medication use (e.g., determine perspectives regarding appropriate medication prescription or deprescribing).

There is currently a gap in that researchers and providers seek to address the priorities of patients, yet often attempt to do so without a clear understanding of these priorities. Potential research questions include: What do people want from their medications? How do they understand the messaging around polypharmacy and deprescribing? What is the need for education to increase people's understanding of medications? Do people selectively take medications and how do they take them? What are the barriers and how can we facilitate communication between patients and providers? How do we encourage shared decision-making? What is the perception of the public and health-care providers about the risks of medications? Do changes in cognition affect patients' values and preferences? Are there cultural differences in communication needs and values/preferences for deprescribing (e.g., new Canadians)? How does end-oflife care (EOL) and advance care planning affect values and preferences? How do patients feel about speaking with other health-care workers (nurses, pharmacist, social worker) about their medications? Would patients be in favour of the reason for the prescription being documented on the prescription? Do patients value issues around the costs of medication?

Priority: Advocacy for clinical trials that specifically evaluate the therapeutic benefit and adverse effects of new and current medications on seniors living with frailty, that evaluate concomitant multiple medication use, and that evaluate the potential for adverse drug-drug interactions specific to seniors living with frailty.

There is complexity in addressing the needs of older frail patients in clinical trials. For example, it is hard to recruit and get frail patients to trial sites, and existing infrastructures may not support this effectively. Although randomized clinical trials are desirable, other data sources should also be utilized to understand the impact of medications on this population. Other metrics for drug impact evaluation should be added, such as abatement of symptoms, reduction in hospital readmissions, and achievement of patients' goals.

Priority: Increasing research into developing or improving models of care that facilitate pharmacists' role in the process of monitoring and assessing use of potentially inappropriate medications.

The group emphasized that patients interact with pharmacists frequently in all settings of care and emphasized the role of a pharmacist as a medication expert. From a research perspective, there is significant evidence that pharmacists can help in many discrete areas of care, but, in the context of frailty, there is less research on the role that the pharmacist can 
play within the larger team to optimize benefit. In developing new models, barriers such as privacy and information needs were discussed, as well as other factors like work flow, communication, and collaborative working relationships to optimize the value of the pharmacist. It was noted that there is evidence available that highlights the benefit of having a pharmacist integrated with the health-care team. This is particularly common in the LTC setting; however, the role of the pharmacist should be explored further in all care settings.

Priority: Improving efforts (e.g., funding) towards developing novel technologies focused on facilitating appropriate medication prescribing and/or deprescribing in older adults living with frailty.

This group reviewed this priority from a broader innovation perspective, where technology could be used to change behaviours to facilitate optimal medication utilization and improved care (e.g., EMRs and web-based tools). Antidepressants were used as an example where it might be possible to offer online cognitive behavioural therapy as an alternative to pharmacological solutions in older adults living with frailty. A caution was that not all frail seniors are willing or are capable of engaging with technology, and this should be considered when developing novel technological innovations. Regardless, the group emphasized there is potential value for this population and that technological innovations should be pursued, developed, and formally evaluated.

\section{Priority: Building an electronic practice system such that a patient's medications can be monitored and reviewed in real time to determine the current need and appropriateness of each medication, as well as potential for adverse drug-drug interactions.}

As a long-term goal, it was suggested that a unified, widely used, well understood, and nationwide electronic system is essential to achieve this priority. The group also recommended the creation of a polypharmacy application for seniors, their families, and caregivers; the application could analyze drugdrug interactions and recommend physician consultation if concerns arose. It should be noted that elements of this technology are already built into many smartphones, some of which have robust health-tracking applications in their operating systems. The banking/finance industry was again used as an example, with the suggestion that Canadians should be able to also have a chip card (like a debit card) which carries personal prescription/health-care data. The group also suggested that the expertise of the programming/ development community serving the finance industry could be leveraged to address health care. Challenges were identified, including confidentiality and ownership particularly when patient-centred care and data are concerned. There is also the challenge of having multiple, incompatible electronic systems across the many jurisdictions of Canadian health care, which has severely limited their penetration into the health-care system across the country.

\section{CONCLUSION AND FINAL RANKING OF PRIORITIES}

The purpose of this Delphi process and summit was to prioritize issues related to medication optimization for older adults living with frailty in the context of the larger CFN mandate and beyond. The participants suggested there are many opportunities to partner with other organizations, which would maximize scope and spread. The top three priorities identified in this process include: i) improving efforts (e.g., funding) towards developing novel innovations focused on facilitating appropriate medication prescribing and/or deprescribing in older adults living with frailty; ii) increasing research into developing or improving models that facilitate pharmacists playing an active role in the process of monitoring and assessing use of potentially inappropriate medications; and iii) encouraging further research into the values and preferences held by elderly patients living with frailty with respect to medication use.

\section{ACKNOWLEDGEMENTS}

Canadian Frailty Network (CFN) is a pan-Canadian network focused on the care of older frail citizens. CFN is comprised of nearly 3,500 corporate and non-profit partners, researchers, scientists, health-care professionals, citizens, students, trainees, educators, and decision-makers. CFN supports and catalyzes original research and innovations to improve the care and quality of life of frail Canadians across all settings of care. The Network also trains the next generation of health-care professionals and scientists. CFN is funded by the Government of Canada through the Networks of Centres of Excellence (NCE) Program. In early 2017, in recognition of the work done in its first five years of operation, the Government of Canada announced funding for a second five-term (2017-2022).

\section{CONFLICT OF INTEREST DISCLOSURES}

The authors declare that no conflicts of interest exist.

\section{REFERENCES}

1. Rotermann M, Sanmartin C, Hennessy D, et al. Prescription medication use by Canadians aged 6 to 79. Health Rep. 2014;25(6):3-9.

2. Ramage-Morin PL. Medication use among senior Canadians. Health Rep. 2009;20(1):37-44.

3. Canadian Institute for Health Information. Drug use among seniors on public drug programs in Canada, 2012. Revised October 2014. Ottawa, ON: CIHI; 2014.

4. Fried TR, O'Leary J, Towle V, et al. Health outcomes associated with polypharmacy in community-dwelling older adults: a systematic review. J Am Geriatr Soc. 2014;62(12):2261-72. 
5. Hamilton $\mathrm{H}$, Gallagher P, Ryan C, et al. Potentially inappropriate medications defined by STOPP criteria and the risk of adverse drug events in older hospitalized patients. Arch Intern Med. 2011;171(11):1013-19.

6. Herr M, Robine JM, Pinot J, et al. Polypharmacy and frailty: prevalence, relationship, and impact on mortality in a French sample of 2350 old people. Pharmacoepidemiol Drug Saf. 2015;24(6):637-46.

7. Bromfield SG, Ngameni CA, Colantonio LD, et al. Blood pressure, antihypertensive polypharmacy, frailty, and risk for serious fall injuries among older treated adults with hypertension. Hypertension. 2017;70(2):259-66.

8. Ballew SH, Chen Y, Daya NR, et al. Frailty, Kidney Function, and Polypharmacy: The Atherosclerosis Risk in Communities (ARIC) Study. Am J Kidney Dis. 2017;69(2):228-36.

9. Veronese N, Stubbs B, Noale M, et al. Polypharmacy Is Associated With Higher Frailty Risk in Older People: An 8-Year Longitudinal Cohort Study. J Am Med Dir Assoc. 2017;18(7):624-28.

10. Saum KU, Schottker B, Meid AD, et al. Is Polypharmacy Associated with Frailty in Older People? Results From the ESTHER Cohort Study. J Am Geriatr Soc. 2017;65(2):e27-e32.

11. Hsu CC, Sandford BA. The Delphi technique: making sense of consensus. Practical Assess Res Evaluation. 2007;12(10):1-8.

12. Sli.do. Slido-Audience Interaction Made Easy [Internet Homepage]. Retrieved from: https://www.sli.do. Accessed 6 July 2017.

13. Canadian Institute for Health Information. National prescription drug utilization information system database — plan information document. 2016. Ottawa, ON: CIHI;2016.

14. Canadian Institute for Health Information. Continuing care metadata [Internet]. Ottawa (ON): CIHI; 2017. Retrieved from: https://www.cihi.ca/en/continuing-care-metadata. Accessed 4 July 2017.

15. Jano E, Aparasu RR. Healthcare outcomes associated with Beers' criteria: a systematic review. Ann Pharmacother. 2007;41(3):438-47.

16. Aparasu RR, Mort JR. Inappropriate prescribing for the elderly: Beers' criteria-based review. Ann Pharmacother. 2000;34(3):338-46.
17. Anderson C. Multinational comparisons of health systems data. The Commonwealth Fund. New York: The Commonwealth Fund; 2014.

18. Live better, together! PatientsLikeMe [Internet homepage]. Retrieved from: https://www.patientslikeme.com/. Accessed 4 July 2017.

19. Proteus Digital Health [Internet homepage]. Retrieved from: www.proteus.com. Accessed 7 July 2017.

20. Evidence-Proteus Digital Health [Internet]. Retrieved from: http://www.proteus.com/evidence/. Accessed 6 July 2017.

21. The Commonwealth Fund. International health policy and practice innovations-The Commonwealth Fund [Internet]. Retrieved from: http:/www.commonwealthfund.org/grantsand-fellowships/programs/international-health-policy. Accessed 6 July 2017.

22. Canadian Deprescribing Network $(\mathrm{CaDeN})$. Deprescribing guidelines and algorithms [Internet]. Retrieved from: http:// deprescribing.org/resources/deprescribing-guidelinesalgorithms/. Accessed 6 July 2017.

23. Budnitz DS, Shehab N, Kegler SR, et al. Medication use leading to emergency department visits for adverse drug events in older adults. Ann Intern Med. 2007;147(11):755-65.

24. Siu HY, Mangin D, Howard M, et al. Developing and testing an electronic medication administration monitoring device for community dwelling seniors: a feasibility study. Pilot Feasibility Stud. 2017;3:5.

25. Bell JS, Airaksinen MS, Lyles A, et al. Concordance is not synonymous with compliance or adherence. $\mathrm{Br} J \mathrm{Clin}$ Pharmacol. 2007;64(5):710-11.

26. Burry L, Mehta S, Williamson DR, et al. Pharmacological interventions for the treatment of delirium in critically ill patients (Protocol). Cochrane Database of Systematic Reviews. 2015; Issue 6. Art. No.: CD011749.

Correspondence to: Peter Aitken, BBM, Partnerships, Development and Government Relations, Canadian Frailty Network, 100 Stuart St., Kingston, ON K7L 2V6, Canada

E-mail: peter@cfn-nce.ca 


\section{APPENDICES}

\section{Appendix 1: First round Delphi Process Survey}

There is little evidence to guide the care of Canadians living with frailty. Care providers of frail older Canadians do not always know if therapies are beneficial, cause harm or are cost-effective. This is particularly true with medications being prescribed to Canadians living with frailty or those in late life. Contributing to the problem is the polypharmacy that many frail elderly experience.

Canadian Frailty Network is hosting a Medication Optimization Summit that will bring together citizens, stakeholders, experts and researchers interested in frailty and medication prescription/de- prescription or appropriate medication prescription in this vulnerable population. As a Summit attendee you have provided CFN with a number of important concerns which have been distilled down to the key issues below.

As preparation for the Medication Optimization Summit we would appreciate your participation in a Delphi process to help prioritize the issues raised by stakeholders and Summit participants. We hope to spend the majority of the Summit considering the top priority issues identified through this Delphi process. Ultimately the findings of the Medication Optimization Summit will assist CFN in prioritizing areas for funding future research or conducting knowledge translation activities in order to improve the care of seniors living with frailty.

Please consider each issue carefully and rank its importance from 1 to 10 (ten being the most important). If there is a follow-on question please provide your answer in the text box provided.

Question 1. Please rate the importance of further research into the values and preferences held by elderly patients living with frailty with respect to medication use (e.g., determine perspectives regarding appropriate medication prescription or deprescribing).

Follow-on question to Q1: Please list any other specific issues that should be addressed in regards to medication prescription when planning for end-of-life care and advance care planning and please indicate in your answer(s) the importance of each issue from 1 to 9 , with nine being most important.

Question 2. Please rate the importance of further research into best practices for shared decision-making with respect to medication use by seniors living with frailty.

Question 3. Please rate the importance of further efforts to develop new tools to aid in shared decision-making whereby care givers, patients and patient's families are intimately involved in appropriate medication prescribing and deprescribing.

Question 4. Please rate the importance of educating all health-care providers (e.g., physicians, pharmacists, personal service workers) so that they are aware of, and understand, the American Geriatrics Society Beers' criteria and list of potentially inappropriate medications for older adults.

Question 5. Please rate the importance of educating older adults, their families and friends (i.e., caregivers) on appropriate medication prescription and the AGS Beers' criteria.

Question 6. Please rate the importance of knowledge mobilization activities designed to improve practice such that prescribing physicians routinely consider the AGS Beers' criteria/list of potentially inappropriate medications for older adults.

Question 7. Please rate the importance of increasing research into developing or improving models to that facilitate a physician's utilization of the AGS Beers' criteria in prescribing and deprescribing potentially inappropriate medications.

Question 8. Please rate the importance of pharmacists playing an active role in the process of monitoring and assessing use of potentially inappropriate medications.

Question 9. Please rate the importance of increasing research into developing or improving models that facilitate pharmacists playing an active role in the process of monitoring and assessing use of potentially inappropriate medications.

Question 10. Please rate the importance of allied health teams playing an active role in the process of monitoring and assessing use of potentially inappropriate medications.

Question 11. Please rate the importance of increasing research into developing or improving models that facilitate allied health teams playing an active role in the process of monitoring and assessing use of potentially inappropriate medications.

Question 12. Please rate the importance of improving efforts (e.g., funding) towards developing novel technologies focused on facilitating appropriate medication prescribing and/or deprescribing in older adults living with frailty.

Question 13. Please rate the importance of building an (electronic) system and practice system so that a patient's medications can be monitored and reviewed in real time to determine the current need and appropriateness of each medication, as well as potential for adverse drug-drug interactions. 
Follow-on question to Q13: Who should have access to this system and who should be ultimately responsible for monitoring the system? Please indicate in your answer(s) its importance from 1 to 9 , with nine being most important issue.

Question 14. Please rate the importance of new technologies being developed so that only professional/licensed care providers (e.g., nurse, physician and/or pharmacist) can monitor and assess a patient's medication use.

Question 15. Please rate the importance of new technologies being developed so that patients (and their trained care providers) are empowered to monitor and assess their own medication use.

Question 16. Please rate the importance of developing technologies designed to increase the ease of medication use in seniors living with frailty since they have unique care needs and challenges (e.g., improve delivery systems).

Question 17. Please rate the importance of developing technologies designed to increase compliance (e.g., dosette devices) in seniors living with frailty since they have unique care needs and challenges.

Question 18. Please rate the importance of promoting and supporting provincial collaborations to collectively negotiate public medication costs (i.e., reduce costs) which would disproportionately benefit older adults in late life since this group is likely to be taking more medications.

Question 19. Please rate the importance of promoting and supporting the development of a nation-wide Pharmacare plan for all Canadians, which would disproportionately benefit older adults in late life since this group is likely to be taking more medications.

Question 20. Please rate the importance of using governmental pharmaceutical funders (e.g., Ontario Trillium Drug Program) to manage medication prescribing or deprescribing in those living with frailty.

Question 21. Please rate the importance of more research into the differential use of medications by seniors living with frailty in different health-care settings (e.g., home care vs. long-term care).

Question 22. Please rate the importance of more research to determine the driving forces (e.g., policy, practice, disease burden) responsible for medication use within different health-care settings (e.g., home care vs. long-term care).

Question 23. Please rate the importance of further research on medication use by seniors living with frailty in different provinces (in similar care settings) and determine the forces responsible for any differences (e.g., policy, practice differences). e.g., Does medication use differ in long-term care facilities depending on the province and if so why?

Question 24. Please rate the importance of advocacy for clinical trials that specifically evaluate the therapeutic benefit and adverse effects of new and current medications on seniors living with frailty.

Question 25. Please rate the importance of advocacy for clinical trials that specifically evaluate concomitant multiple medication use and the potential for adverse drug-drug interactions specific to seniors living with frailty.

Question 26. Please rate the importance that all new registration trials for medications targeted at older individuals include and report on the differential impact on individuals living with frailty.

Question 27. If there are concerns or issues not covered by the above, please indicate these in the text box and also assign levels of importance from 1 to 9 , with 9 being the most important issue. 